\title{
ARTICLE
}

Translational Therapeutics

\section{The RNA polymerase I transcription inhibitor CX-5461 cooperates with topoisomerase 1 inhibition by enhancing the DNA damage response in homologous recombination- proficient high-grade serous ovarian cancer}

Shunfei Yan ${ }^{1}$, Jiachen Xuan ${ }^{1}$, Natalie Brajanovski ${ }^{2}$, Madeleine R. C. Tancock ${ }^{3}$, Piyush B. Madhamshettiwar ${ }^{4}$, Kaylene J. Simpson $^{1,4}$,

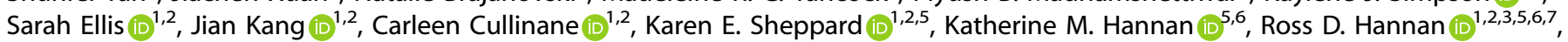
Elaine Sanij $\mathbb{D}^{1,2,8}$, Richard B. Pearson $\mathbb{D}^{1,2,3,5}$ and Keefe T. Chan $\mathbb{1 D}^{1,2}$

BACKGROUND: Intrinsic and acquired drug resistance represent fundamental barriers to the cure of high-grade serous ovarian carcinoma (HGSC), the most common histological subtype accounting for the majority of ovarian cancer deaths. Defects in homologous recombination (HR) DNA repair are key determinants of sensitivity to chemotherapy and poly-ADP ribose polymerase inhibitors. Restoration of HR is a common mechanism of acquired resistance that results in patient mortality, highlighting the need to identify new therapies targeting HR-proficient disease. We have shown promise for CX-5461, a cancer therapeutic in early phase clinical trials, in treating HR-deficient HGSC.

METHODS: Herein, we screen the whole protein-coding genome to identify potential targets whose depletion cooperates with CX5461 in HR-proficient HGSC.

RESULTS: We demonstrate robust proliferation inhibition in cells depleted of DNA topoisomerase 1 (TOP1). Combining the clinically used TOP1 inhibitor topotecan with CX-5461 potentiates a G2/M cell cycle checkpoint arrest in multiple HR-proficient HGSC cell lines. The combination enhances a nucleolar DNA damage response and global replication stress without increasing DNA strand breakage, significantly reducing clonogenic survival and tumour growth in vivo.

CONCLUSIONS: Our findings highlight the possibility of exploiting TOP1 inhibition to be combined with CX-5461 as a nongenotoxic approach in targeting HR-proficient HGSC.

British Journal of Cancer (2021) 124:616-627; https://doi.org/10.1038/s41416-020-01158-z

\section{BACKGROUND}

High-grade serous ovarian carcinoma (HGSC) is the most prevalent histological subtype of epithelial ovarian cancer with the worst prognosis. HGSC is characterised by nearly universal TP53 mutations (>96\%), and $50 \%$ of HGSC harbour defects in homologous recombination (HR) DNA repair genes, the most common alterations occurring in breast cancer-related antigen 1 (BRCA1) and BRCA2, which are necessary for maintaining genomic integrity. ${ }^{1}$ Defects in HR confer exquisite sensitivity of HGSC to standard DNA-damaging chemotherapies (carboplatin/cisplatin and paclitaxel) as well as the recently Food Drug Administrationapproved poly-ADP ribose polymerase (PARP) inhibitors (olaparib, rucaparib, niraparib, talazoparib), which are synthetic lethal by generating catastrophic DNA damage and cell death in cells lacking $\mathrm{HR}^{2}{ }^{2}$ However, resistance to these therapies frequently develops via multiple mechanisms, including stabilisation of stalled DNA replication forks, increased drug efflux, decreased PARP trapping and restoration of HR, a common form of resistance observed in the clinic that inevitably results in patient mortality. ${ }^{3-7}$ Of the $50 \%$ primary HGSC tumours that are HRproficient, 20\% harbour CCNE1 amplifications, which are associated with intrinsic platinum-based chemotherapy, PARPinhibitor resistance and poor clinical outcome. ${ }^{8}$ Therefore, new therapies targeting HR-proficient HGSCs arising from both intrinsic and acquired resistance are urgently needed to improve patient outcomes.

Increased activation of key oncogenic signalling pathways (PI3K/AKT, RAS/MAPK and MYC) upstream of ribosome biogenesis

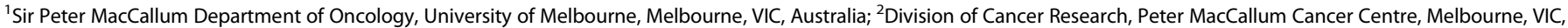

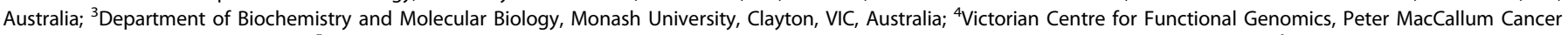

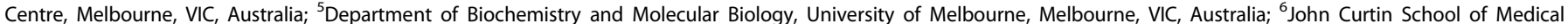

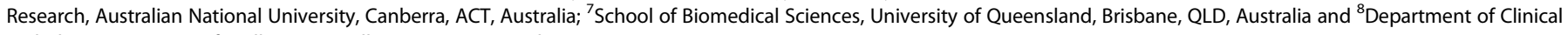
Pathology, University of Melbourne, Melbourne, VIC, Australia

Correspondence: Elaine Sanij (elaine.sanij@petermac.org) or Richard B. Pearson (Rick.Pearson@petermac.org) or Keefe T. Chan (keefe.chan@petermac.org)

These authors contributed equally: Elaine Sanij, Richard B. Pearson, Keefe T. Chan

Received: 10 November 2019 Revised: 5 October 2020 Accepted: 22 October 2020

Published online: 11 November 2020 
constitutes an additional hallmark of $\mathrm{HGSC}^{9}$ and we hypothesise that inhibiting ribosome biogenesis can provide an effective cancer therapeutic option. ${ }^{10}$ Indeed, we have shown encouraging responses with the ribosomal RNA gene (rDNA) transcription inhibitor CX-5461, which inhibits the production of the major ribosomal RNA (rRNA) components of the ribosome, in a Phase I clinical trial in haematological malignancies. ${ }^{11}$ We observed beneficial responses in patients who were wild type (five out of eight) or mutant (one out of five) for TP53, highlighting CX-5461's p53-dependent and -independent therapeutic activity, and a favourable toxicity profile. Furthermore, clinical trials in advanced solid tumours are ongoing, ${ }^{12}$ emphasising the potential promise of CX-5461 in treating a broad range of cancers.

Mechanistically, we have demonstrated in cells with intact p53 that CX-5461 induces an impaired ribosome biogenesis checkpoint leading to apoptosis, cell cycle arrest or senescence. ${ }^{10,13,14}$ However, in cells with inactivated p53 such as HGSC, CX-5461 induces replication stress and the DNA damage response (DDR), leading to a G2/M cell cycle checkpoint arrest in HR-proficient cells and cell death in HR-deficient cells. ${ }^{15}$ Furthermore, combining PARP inhibitors with CX-5461 enhanced cytotoxicity and therapeutic benefit in HR-deficient HGSC models in vitro and in vivo. ${ }^{15}$ CX-5461 has a distinct sensitivity profile compared to PARP inhibitors involving meiotic recombination 11 (MRE11)-dependent degradation of replication forks. These findings highlight the potential of identifying combination therapies to improve the efficacy of CX-5461 in targeting HGSC.

In this report, we performed a whole protein-coding genome RNA interference (RNAi) screen to identify potential targets whose inhibition can enhance the efficacy of CX-5461 in treating HRproficient HGSC. We demonstrate the CX-5461 exhibits a unique sensitivity pattern distinct to those reported for G-quadruplex stabilisers and topoisomerase 2 (TOP2) poisons previously thought to function in an equivalent mechanism to CX-5461. ${ }^{16}$ Importantly, we find that DNA topoisomerase I (TOP1) inhibition can be combined with CX-5461 to target HR-proficient HGSC cells. TOP1 has been shown to localise to rDNA to release torsional stress during transcription and DNA replication of the highly repetitive and transcribed rDNA repeats. ${ }^{17,18}$ We demonstrate that the combination of the TOP1 inhibitor topotecan and CX-5461 exacerbates replication stress at the rDNA repeats and across the genome. We show that the combination of CX-5461 and topotecan inhibits proliferation of HR-proficient HGSC by enhancing G2/M checkpoint arrest induced by replication stress and activation of the ATR pathway without further generating DNA strand breaks compared to single-agent treatment. Furthermore, the combination of CX-5461 and topotecan leads to significantly improved regression of HR-proficient HGSC tumours in vivo, highlighting the combination as a promising approach for treating HR-proficient HGSC.

\section{Methods}

Cell culture. Human HGSC cell lines (OVCAR4, OVCAR3 and CAOV3) were obtained from the National Cancer Institute. All cell lines were short tandem repeat (STR) characterised against American Tissue Type Collection or ExPASy databases to ensure the authenticity of origin. Mycoplasma tests were performed routinely by PCR. All cells were cultured in RPMI-1640 media (Gibco) supplemented with $10 \%$ foetal bovine serum (FBS) (SigmaAldrich) and $2 \mathrm{mM}$ GlutaMax ${ }^{\mathrm{TM}}$ (Gibco) at $37^{\circ} \mathrm{C}$ and $5 \% \mathrm{CO}_{2}$.

Generation of inducible BRCA2 knockdown cell lines. The pLKO TetOn construct expressing a doxycycline-inducible short hairpin RNA (shRNA) targeting BRCA2 (GGGAAACACUCAGAUUAAA) was a kind gift from Madalena Tarsounas. ${ }^{19}$ OVCAR4 cells were transduced with VSVG-pseudotyped lentivirus produced in HEK$293 \mathrm{~T}$ cells and $4 \mu \mathrm{g} / \mu \mathrm{L}$ polybrene, and cells were selected with 1 $\mu \mathrm{g} / \mathrm{mL}$ puromycin for 3 days prior to use in experiments.
Generation of TOP1 knockout cell lines. OVCAR4 and OVCAR3 cells $\left(3 \times 10^{5}\right)$ in $20 \mu \mathrm{L}$ SF Cell Line Nucleofector ${ }^{\mathrm{TM}}$ Solution (Lonza) were nucleofected with ribonucleoprotein complexes containing $3.4 \mu \mathrm{L}$ phosphate-buffered saline (PBS), $0.6 \mu \mathrm{L}$ Alt- $\mathrm{R}^{\circledR}$ S.p. Cas9 nucleasepurified Cas9 protein (Integrated DNA Technologies) and $1 \mu \mathrm{L}$ of $300 \mu \mathrm{M}$ chemically modified EZ scaffold (Synthego) control single guide RNA (sgRNA) (CAUUUCUCAGUGCUAUAGAG) or 0.5 $\mu \mathrm{L}$ of each TOP1 sgRNA (sgRNA \#1: ACUCACUCAUCCUCAUCUCG; sgRNA \#2: CAAACAUAAAGACAGAGACA) using a 4D-Nucleofector $^{\text {TM }}$ (Lonza).

Reagents and antibodies. CX-5461 was provided by SYNkinase and prepared in $50 \mathrm{mM} \mathrm{NaH} \mathrm{PO}_{4}$. Topotecan (Hycamtin ${ }^{\circledR}$, Novartis) was obtained from the Peter Mac pharmacy and was dissolved in $0.9 \%$ saline. $\mathrm{DBL}^{\mathrm{TM}}$ doxorubicin hydrochloride injection was purchased from Hospira and diluted in PBS. The pan-caspase inhibitor Q-VD-OPh (Cat. \#1901) was purchased from APExBIO and dissolved in dimethyl sulfoxide. A list of antibodies used in this study is provided in Supplementary Table S1.

Genome-wide protein-coding RNAi primary screen and analysis. On a screening day, $160 \mu \mathrm{L}$ DharmaFECT 4 (Horizon Discovery) was mixed with $50 \mathrm{~mL}$ Opti-MEM $^{\circledR}$ (Gibco) (sufficient for 16 assay plates), and $44 \mu \mathrm{L}$ lipid:Opti-MEM mixture was then aliquoted to each well of a 384-well black-walled plate (Corning, Cat. \#3712) containing $6 \mu \mathrm{L}$ of $1 \mu \mathrm{M}$ SMARTpool small interfering RNA (siRNA) (Horizon Discovery) using a BioTek EL406 ${ }^{\mathrm{TM}}$ washer dispenser (final SMARTpool concentration $40 \mathrm{nM}$ ). The transfection mixture was mixed and complexed for $20 \mathrm{~min}$, and $12.5 \mu \mathrm{L}$ mixture was then aliquoted into three plates (four replicate plates in total) using a Caliper Sciclone ALH3000 liquid handler. During this period, OVCAR4 cells were trypsinised and resuspended at $5.6 \times 10^{4}$ cells/ $\mathrm{mL}$. Twenty-five microlitres of OVCAR4 cells (1400 cells) was then dispensed into 384-well plates with the final concentration of the SMARTpool siRNA at $40 \mathrm{nM}$, and plates were incubated at $37^{\circ} \mathrm{C}$ and $5 \% \mathrm{CO}_{2}$. At $24 \mathrm{~h}$ post transfection, the medium was replaced

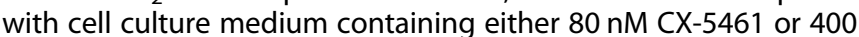
$\mathrm{nM} \mathrm{NaH} \mathrm{PO}_{4}$. Cells were incubated for another $48 \mathrm{~h}$ and fixed with $2 \%$ paraformaldehyde in PBS for $10 \mathrm{~min}$, followed by permeabilisation with $0.3 \%$ Triton $\mathrm{X}-100$ in PBS for $10 \mathrm{~min}$. Cells were washed with PBS, stained with $100 \mathrm{ng} / \mathrm{mL}$ 4',6-diamidino-2phenylindole (DAPI) for $20 \mathrm{~min}$. Cells were washed twice with PBS and 25 fields were imaged using the ArrayScan VTI highcontent system (Thermo Fisher Scientific) with a $\times 20 / 0.4$ NA objective and an ORCA-ER camera and a 5-ms exposure time. The Cellomics Morphology V4 Bioapplication was used to analyse cell number as determined by DAPI staining.

To identify synergistic gene candidates, we used a combination of the difference in relative cell number between vehicle- and CX5461-treated target siRNA plates normalised to ON-TARGETplus (Horizon Discovery) non-targeting control (siControl) $\geq 0.25$, and a value for the coefficient of drug interaction $(C D I) \leq 0.9$, which was calculated based on a Bliss independence model: ${ }^{20}$

$\mathrm{CDI}=($ relative cell number $($ siRNA $+C X-5461)) /($ relative cell number $($ siRNA + vehicle) $\times$ relative cell number $($ siControl $+C X$ 5461)).

Secondary deconvolution screen. Based on the above criteria, we selected 372 genes for a secondary deconvolution screen using the four individual siRNA duplexes that comprised the SMARTpool arrayed separately (1 duplex/well). For the secondary screen, the 1 $\mu \mathrm{M}$ SMARTpool siRNAs were replaced by $0.45 \mu \mathrm{M}$ individual siRNA duplexes for a final concentration of $25 \mathrm{nM}$ /duplex. We modified our selection criteria to define high-confidence hits as those with a difference in relative cell number between vehicle- and CX-5461treated target siRNA plates normalised to siControl $\geq 0.15$ and Bliss independence $\leq 0.8$. We classified genes with $\geq 2$ high-confidence hits as those displaying synergy. 
Quantitative real-time $P C R$. The RNeasy ${ }^{\circledR}$ Mini Kit (Qiagen) was used to extract total RNA as per the manufacturer's instructions. One hundred nanograms of total RNA was used as a template for CDNA synthesis using SuperScript ${ }^{\mathrm{TM}}$ III reverse transcriptase (Invitrogen ${ }^{\mathrm{TM}} \# 18080093$ ), hexameric random primers and dNTPs. Quantitative real-time PCR (qPCR) reactions were performed using Fast SYBR $^{\otimes}$ Green reagents in a StepOnePlus ${ }^{\text {tM }}$ Real-Time PCR system (Applied Biosystems ${ }^{T M}$ ) with a $+0.7^{\circ} \mathrm{C}$ melt increment. RNase-free water was used as a negative control. Changes in target gene expression were normalised to NONO housekeeping gene and fold change was determined by using $2 \wedge\left(-\Delta \Delta C_{t}\right)$. Primer sequences are listed in Supplementary Table S2.

Immunoblotting. Cells were washed twice with PBS and wholecell lysates were prepared in Western solubilisation buffer $(20 \mathrm{mM}$ HEPES pH 7.9, 2\% sodium dodecyl sulfate, 0.5 mM EDTA). Twenty micrograms of protein was transferred to polyvinylidene fluoride membranes, which were blocked in $5 \%$ skim milk TBS $0.1 \%$ Tween $^{\circledR} 20$ (TBST) for $45 \mathrm{~min}$ at room temperature (RT). Membranes were incubated with primary antibodies overnight at $4{ }^{\circ} \mathrm{C}$, washed three times in TBST for $10 \mathrm{~min}$, incubated with horseradish peroxidase-conjugated secondary antibodies for $1 \mathrm{~h}$ at RT and then washed. Membranes were visualised using Western Lightning $^{\text {TM }}$ Plus enhanced chemiluminescence (PerkinElmer) by exposure to film (Fujifilm SuperRX) or imaged by a ChemiDoc ${ }^{\mathrm{TM}}$ Touch Imaging System (Bio-Rad Technology). Digital scans of film were acquired using an Epson Perfection V700 Photo at $\geq 300 \mathrm{dpi}$ (dots per inch).

Immunofluorescence. Five thousand cells were seeded into 8-well Nunc $^{\text {TM }}$ Lab-Tek $^{\mathrm{TM}}$ II Chamber Slides ${ }^{\mathrm{TM}}$ (Cat. \#154534) per well. Cells were cultured for $72 \mathrm{~h}$, followed by drug treatments at the indicated timepoints and doses together with $10 \mu \mathrm{M}$ 5-ethynyl-2'deoxyuridine (EdU) (Sigma-Aldrich, Cat. \#900584). Cells were fixed in $4 \%$ paraformaldehyde and permeabilised with $0.5 \%$ Triton X100 in PBS. EdU was fluorescently labelled with $0.5 \mu \mathrm{M}$ Click-iT ${ }^{\mathrm{TM}}$

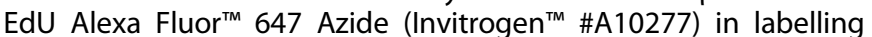
buffer (100 mM Tris, pH 8.5, $100 \mathrm{mM}$ ascorbic acid, 1 mM CuSO ${ }_{4}$ ) for $30 \mathrm{~min}$ at RT and subsequently stained with the indicated antibodies. Cell nuclei were stained with $500 \mathrm{ng} / \mathrm{mL}$ DAPI diluted in PBS. Slides were mounted with Vectashield ${ }^{\circledR}$ Antifade Mounting Media (Vector Laboratories, Cat. \#H-1000). Images were captured by a Zeiss LSM 780 confocal microscope using a $\times 20 / 0.8$ NA Planapochromat objective and were analysed with FIJI $\mathrm{v} 1.52 \mathrm{p}^{21}$ and CellProfiler v3.1.19. ${ }^{22}$

Cell cycle analysis. Cells were labelled with $10 \mu \mathrm{M}$ 5-bromo-2'deoxyuridine (BrdU) (Thermo Fisher Scientific, Cat. \#B23151) for 30 min, collected with supernatant and fixed with ice-cold $80 \%$ ethanol. Fixed cells were centrifuged, resuspended in $2 \mathrm{~N} \mathrm{HCl}+$ $0.5 \%$ Triton $\mathrm{X}-100(\mathrm{v} / \mathrm{v})$ and acid was neutralised with $0.1 \mathrm{M}$ $\mathrm{Na}_{2} \mathrm{~B}_{4} \mathrm{O}_{7} \cdot 10 \mathrm{H}_{2} \mathrm{O}(\mathrm{pH} 8.5)$. Cells $\left(2 \times 10^{5}\right)$ were incubated with 100 $\mu \mathrm{L}$ anti-BrdU antibody $(0.5 \mu \mathrm{g} / \mathrm{mL}$ ) in dilution buffer (PBS $+2 \%$ FBS) $+0.5 \%$ Tween-20 for $30 \mathrm{~min}$ at room temperature, washed with dilution buffer and resuspended in $100 \mu \mathrm{L}$ in Alexa Fluor 488 donkey anti-mouse $\operatorname{lgG}(5 \mu \mathrm{g} / \mathrm{mL})$ in dilution buffer $+0.5 \%$ Tween20 for $30 \mathrm{~min}$ on ice and then then washed. Cells were resuspended in $10 \mu \mathrm{g} / \mathrm{mL}$ propidium iodide (PI) (Sigma-Aldrich, Cat. \#P4170) in dilution buffer and analysed by flow cytometry on a BD FACSCanto ${ }^{\mathrm{TM}}$ II. Quantitation of cell cycle populations was performed using the FlowJo v9.3.2 software.

Clonogenic assay. Cells $\left(1 \times 10^{4} /\right.$ well $)$ were seeded into 6 -well plates (BD Falcon). Cells were cultured for $24 \mathrm{~h}$, and then drugged as indicated. At $48 \mathrm{~h}$ after drug treatment, the drugs were removed by washing twice with PBS. Cells were then cultured in normal medium for another 5 days when the vehicle-treated cells reached confluency. Cells were then fixed with $100 \%$ methanol for
$10 \mathrm{~min}$, stained with $0.1 \%$ crystal violet solution for $20 \mathrm{~min}$, thoroughly washed with PBS and the stained plates were dried, imaged on an IncuCyte ${ }^{\circledR}$ ZOOM (Essen Bioscience) using a $\times 10 / 0.3$ NA objective and analysed for cell confluence.

DNA fibre analysis. DNA fibre analysis was performed using methods as those previously described. ${ }^{15}$ OVCAR4 cells $\left(1 \times 10^{6}\right)$ were plated in $10-\mathrm{cm}$ dishes for $24 \mathrm{~h}$. The medium was replaced with culture medium containing $50 \mu \mathrm{M}$ 5-chloro-2-deoxyuridine (CldU) and cells were incubated at $37{ }^{\circ} \mathrm{C} 5 \% \mathrm{CO}_{2}$ for $30 \mathrm{~min}$. Cells were washed three times with PBS, and fresh pre-warmed medium containing $250 \mu \mathrm{M}$ 5-iodo- $2^{\prime}$-deoxyuridine (IdU) was added and cells were incubated at $37^{\circ} \mathrm{C} 5 \% \mathrm{CO}_{2}$ for $30 \mathrm{~min}$. Cells were washed three times with warm PBS and treated as indicated for $3 \mathrm{~h}$ at $37^{\circ} \mathrm{C} 5 \% \mathrm{CO}_{2}$. Labelled cells were trypsinised, pelleted, washed twice with PBS and resuspended in a pre-warmed solution $\left(50^{\circ} \mathrm{C}\right)$ composed of PBS with $0.05 \%$ phenol red-free trypsin. Cellular suspensions were then mixed carefully with an equal volume of $1.2 \%$ low melt agarose (Bio-Rad, 1613111), and the mixture was dispensed into a plug mould (Bio-Rad, 1703713) and allowed to set at $4{ }^{\circ} \mathrm{C}$ for $1 \mathrm{~h}$. Solidified plugs were then pushed out of the mould and transferred into 2-mL polypropylene tubes containing 0.5 M EDTA pH 8, 1\% (v/v) Sarkosyl (Sigma-Aldrich, 61743), and proteinase K (Roche Applied Science, 3115828001). The agarose plugs were incubated in this buffer at $50{ }^{\circ} \mathrm{C}$ overnight. Following incubation, agarose plugs were washed extensively $(1 \mathrm{M}$ Tris, 0.1 M EDTA pH 8) and digested with $\beta$-agarase (New England Biolabs, M0392S) overnight in 0.5 M MES hydrate (Sigma-Aldrich, M5287) $\mathrm{pH} 5.5$ at $42{ }^{\circ} \mathrm{C}$. Samples were then poured carefully into a FiberComb reservoir (Genomic Vision, RES-001) and the DNA solution combed onto silanised coverslips (Genomic Vision, COV002) at a constant speed of $250 \mu \mathrm{m} / \mathrm{s}$, using the Molecular Combing System by Genomic Vision (MCS-001). For easy handling following combing, coverslips were adhered to glass SuperFrost ${ }^{\circledast}$ Plus slides (Menzel Gläser) using cyanoacrylate glue and then baked in an incubator at $65^{\circ} \mathrm{C}$ for $2 \mathrm{~h}$ to irreversibly crosslink DNA to the surface. Next, samples were immersed in a solution containing $0.5 \mathrm{M} \mathrm{NaOH}$ and $1 \mathrm{M} \mathrm{NaCl}$ for $8 \mathrm{~min}$ at room temperature to denature the combed DNA, before being washed thoroughly with PBS. Coverslips were subsequently dehydrated by incubating them in increasing concentrations of $70 \%, 90 \%$ and $100 \%$ of ethanol for $5 \mathrm{~min}$ each, followed by air drying. To minimise non-specific binding of antibodies, blocking buffer containing $0.1 \%$ Triton $\mathrm{X}-100$ and $5 \%$ bovine serum albumin was applied to all slides for $30 \mathrm{~min}$ at room temperature prior to staining. DNA fibres attached to coverslips were then probed with rat anti-BrdU antibody (1:100, Abcam ab6323) specific to CldU and mouse anti-BrdU antibody (1:50, Becton Dickinson, 347580) specific to IdU for $1 \mathrm{~h}$ at $37^{\circ} \mathrm{C}$ in a humidified chamber. Following staining, slides were washed three times with PBS and subsequently incubated with Alexa Fluor 488-conjugated goat anti-rat antibody (Invitrogen, A-11006) and Alexa Fluor 594-labelled donkey anti-mouse antibody (Invitrogen, A-21203) at 1:200 dilutions, for $1 \mathrm{~h}$ at $37^{\circ} \mathrm{C}$. Lastly, slides were washed three times in PBS, mounted and visualised using the Nikon C2 confocal microscopy at $\times 40$ magnification. Images were taken of 100 fibres per condition. Only high-quality and well-separated DNA fibres (not entangled DNA regions) were measured using ImageJ software $(1.47 \mathrm{v}, \mathrm{NIH})$. The ratio of IdU to $\mathrm{CldU}$ tracks in each fibre was calculated and graphed using GraphPad Prism 8.

DNA comet assay. DNA comet assays were performed using the CometAssay ${ }^{\circledR}$ Reagent Kit (Trevigen, Cat. \#4250-050-K) according to the manufacturer's protocol. Briefly, cells treated with indicated drugs were trypsinised and washed once with ice-cold PBS, and then resuspended in ice-cold PBS at $1 \times 10^{5}$ cells $/ \mathrm{mL}$. Cells were mixed with molten low-melt agarose at $37^{\circ} \mathrm{C}$ at a ratio of $1: 10$ and $50 \mu \mathrm{L}$ of the mixture was immediately pipetted onto a 
CometSlide ${ }^{\mathrm{TM}}$. The slides were incubated in the dark at $4{ }^{\circ} \mathrm{C}$ for 30 min to solidify the agarose and immersed in $4^{\circ} \mathrm{C}$ lysis solution overnight. Slides were then immersed in freshly prepared alkaline unwinding solution for $1 \mathrm{~h}$ at $4^{\circ} \mathrm{C}$ in the dark, and electrophoresed in $4{ }^{\circ} \mathrm{C}$ alkaline electrophoresis solution at $300 \mathrm{~mA}$ for $40 \mathrm{~min}$. The slides were washed with $\mathrm{ddH}_{2} \mathrm{O}$ twice, followed by $70 \%$ ethanol for $5 \mathrm{~min}$. Slides were dried at $37^{\circ} \mathrm{C}$ for $15 \mathrm{~min}$, and then stained with $2.5 \mu \mathrm{g} / \mathrm{mL}$ PI in PBS for $30 \mathrm{~min}$ at room temperature. Slides were rinsed twice in $\mathrm{dd}_{2} \mathrm{O}$ and completely dried at $37^{\circ} \mathrm{C}$. Images were captured using a VS120 Virtual Slide Microscope (Olympus) using a $\times 10$ objective and analysed with the OpenComet v1.3.1 23 plugin for ImageJ 1.51 .

Xenograft transplantation. All animal studies were conducted according to the protocols approved by the Animal Experimentation Ethics Committee at the Peter MacCallum Cancer Centre. Non-obese diabetic severe-combined immunodeficiency gamma (NSG) mice (6-8 weeks old female) were purchased from the Garvan Institute (Australian BioResources) and housed in animal cages under standard laboratory conditions. OVCAR3 cells $\left(6 \times 10^{6}\right)$ in $100 \mu \mathrm{L}$ ice-cold PBS:Matrigel (1:1) were subcutaneously injected into the right flank of mice anaesthetised with isoflurane. Tumour-bearing mice were weighed and measured twice weekly with callipers. Once tumours reached an average volume of $100 \mathrm{~mm}^{3}$, mice were randomised into four groups of ten mice. Mice were dosed with vehicle $(25 \mathrm{mM}$ $\left.\mathrm{NaH}_{2} \mathrm{PO}_{4}\right)$ or CX-5461 $(30 \mathrm{mg} / \mathrm{kg})$ via oral gavage twice weekly, while topotecan $5 \mathrm{mg} / \mathrm{kg}$ or vehicle $(0.9 \%$ saline) was delivered via intraperitoneal injection twice weekly. Mice were dosed for 4 consecutive weeks for a total of eight doses. Bodyweight and tumour volumes were monitored daily during and after the treatment period. Mice were euthanised by cervical dislocation upon reaching any ethical endpoint of the experiment (signs of distress or tumour volume $\geq 1200 \mathrm{~mm}^{3}$ ).

Statistical analysis software. Prism 8 (GraphPad) was used for all the statistical analyses as indicated, including dose-response curves, two-sided $t$ tests, Wilcoxon's rank-sum non-parametric tests, ordinary one-way analysis of variance (ANOVA), Kruskal-Wallis non-parametric one-way ANOVA and Mantel-Cox tests. Synergy was quantified using Combenefit v2.02. ${ }^{24}$

\section{RESULTS}

A functional genomics screen identifies a network of genes that when depleted cooperates with CX-5461 to inhibit HR-proficient HGSC cell proliferation

To identify genes that, when depleted, could synergise with CX5461 to inhibit HR-proficient HGSC cell proliferation, we used human OVCAR4 cells $^{25}$ in a protein-coding genome-wide RNAi screen (Fig. 1a). Based on quantifying the reduction in cell number relative to control siRNA (siControl) and the CDI to assess combinatorial effects, we identified 372 genes whose knockdown demonstrated synergy with CX-5461 (Table S3). Gene ontology analysis of these candidates identified enrichment in several functional processes, with DNA damage:double-strand break (DSB) repair being the most significant (Fig. $1 \mathrm{~b}$ and Table S4). Furthermore, analysis of the gene candidates involved in different DNA repair pathways showed a unique sensitivity pattern distinct to those reported for pyridostatin, a G-quadruplex stabiliser, and TOP2 poisons thought to function via an equivalent mechanism to CX-5461 (Fig. 1c and Table S5). ${ }^{16,26}$ Deconvolution screening using individual siRNA duplexes and more stringent metrics validated 20 genes using a concordance criterion of two out of four (Fig. 1d and Table S6). STRING network analysis demonstrated significant enrichment of genes involved in the HR pathway (Fig. 1e), including BRCA2 (4/4 siRNA duplexes validated). To further confirm this finding, we generated OVCAR4 cells expressing a doxycycline- inducible BRCA2 shRNA (Fig. S1A). ${ }^{19}$ Treating OVCAR4 cells depleted of BRCA2 with CX-5461 led to a two-fold reduction in cell proliferation (Fig. S1B), which is consistent with the magnitude of reduction previously reported in the PEO1 ovarian cancer cell line harbouring BRCA2 knockout. ${ }^{26}$ Together, these data indicate that inducing HR deficiency in HR-proficient cells can enhance their sensitivity to CX-5461.

Of the validated candidates, we also identified TOP1 (2/4 siRNA duplexes validated) (Fig. 1f), whose physiological function is to relieve DNA supercoiling that occurs during transcription and replication by generating single-strand breaks. ${ }^{17}$ To further validate this result, we knocked out TOP1 in OVCAR4 cells using clustered interspaced short palindromic repeats/Cas9-mediated gene editing (Fig. S1C). Clonogenic assays showed significantly reduced colony formation in TOP1-knockout cells treated with CX5461 compared to control (Fig. S1D). Knockout of TOP1 in another HR-proficient HGSC cell line OVCAR3 (Fig. S1E) also resulted in decreased colony formation (Fig. S1F) upon treatment with CX5461 compared to control. Collectively, these data suggest that TOP1 depletion cooperates with CX-5461 in inhibiting HRproficient HGSC cell proliferation.

The combination of CX-5461 and topotecan induces a robust G2/ $M$ cell cycle checkpoint arrest

The TOP1 inhibitor topotecan is used in salvage therapy for recurrent $\mathrm{HGSC}^{27}$ with a Phase III clinical trial showing $29 \%$ and $6.5 \%$ objective response rates in platinum-sensitive and -refractory patients, respectively. ${ }^{28}$ Given that we observed combinatorial effects between CX-5461 and TOP1 depletion, we reasoned that CX-5461 could extend the utility of TOP1 inhibitor topotecan to target HR-proficient HGSC. We performed dose-response proliferation assays of three HR-proficient cell lines (OVCAR4, OVCAR3 and CAOV3) treated with CX-5461 or topotecan (Fig. S2A, B) to determine appropriate concentrations for comparison in drug checkerboard assays. Drug checkerboard assays demonstrated synergistic suppression of proliferation in all three cell lines (Fig. 2a).

To determine the cellular response contributing to the ability of the combination of CX-5461 and topotecan to inhibit cell proliferation, we performed cell cycle analyses of OVCAR4, OVCAR3 and CAOV3 cells. While treatment with CX-5461 or topotecan led to a trend toward an increase in G2/M cell cycle arrest compared to control, we observed significant enrichment in cells arresting in $\mathrm{G} 2 / \mathrm{M}$ with the combination (Fig. $2 \mathrm{~b}$ and Fig. S3A). In addition, we performed qPCR of CDKN2A (Fig. S3B) and CDKN1A (Fig. S3C), encoding for the cyclin-dependent kinase inhibitors p16 and p21, respectively, in OVCAR4 cells treated with vehicle, CX5461 , topotecan or the combination. We found that p21 but not p16 was induced in the combination, supporting p53independent induction of p21 during the G2/M cell cycle arrest. We also examined the sub-G1 population to determine the impact of the treatments on cell death. OVCAR4 and CAOV3 but not OVCAR3 cells showed a significant increase in the sub-G1 population with combined CX-5461 and topotecan compared to control (Fig. S3D). The increase in cell death upon combined treatment was partially rescued by the caspase inhibitor Q-VD$\mathrm{OPh}$, implicating a role for apoptosis. Taken together, these data demonstrate that the combination of CX-5461 and topotecan can, at least in part, promote cell death but predominantly induces cell cycle arrest in HR-proficient HGSC cells.

Combined treatment of CX-5461 and topotecan potentiates the DDR

We previously showed the p53-independent response to CX-5461 is mediated by ataxia telangiectasia mutated (ATM) and ATM and Rad3-related (ATR), both of which are critical regulators of the DDR pathway ${ }^{15,29}$ and are activated by various forms of DNA damage ${ }^{30}$; hence, we hypothesised that the DDR would be a key mediator of 

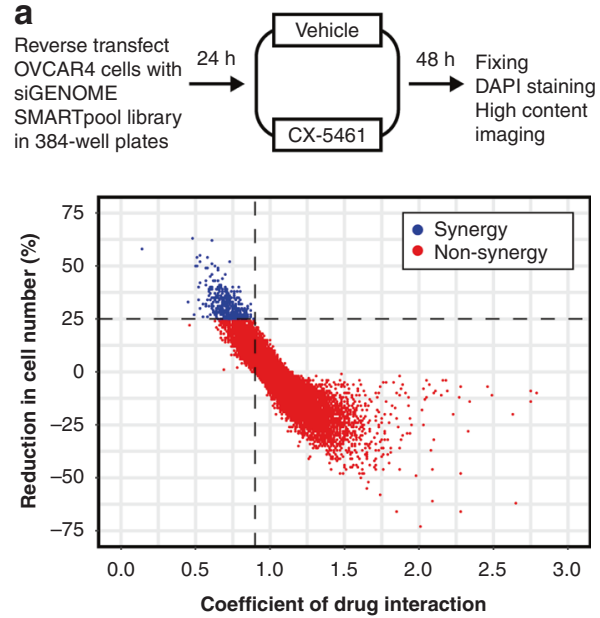

b

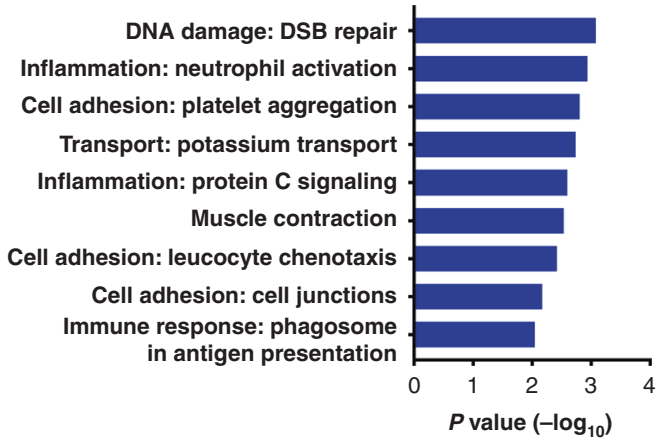

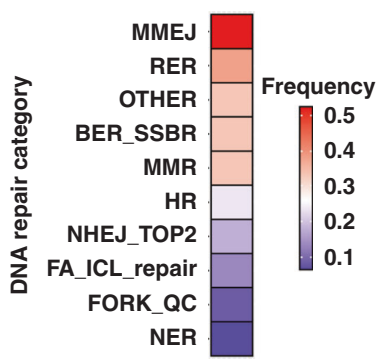

d

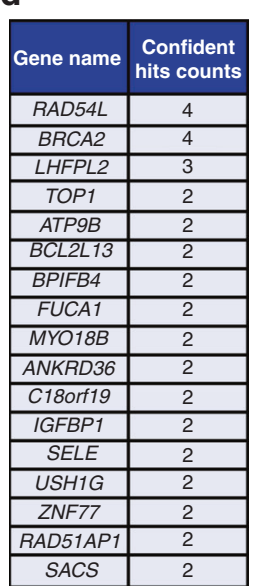

e

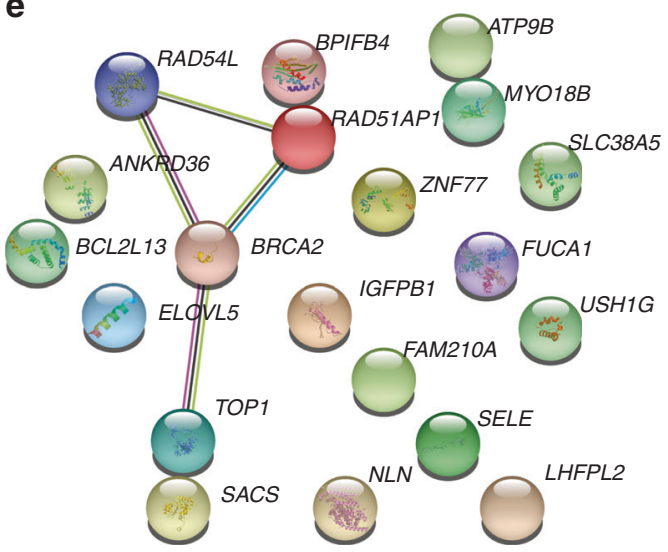

f

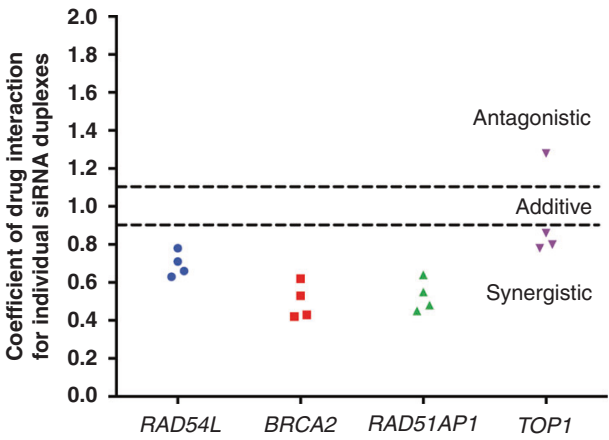

Fig. 1 A genome-wide RNAi screen identifies a network of genes that when depleted synergise with CX-5461 in inhibiting HGSC cell proliferation. a Schematic representation of the genome-wide primary screen design. OVCAR4 cells were reverse transfected with the siGENOME SMARTpool library. After $24 \mathrm{~h}$, cells were treated with vehicle control $\left(400 \mathrm{nM} \mathrm{NaH}_{2} \mathrm{PO}_{4}\right)$ or $80 \mathrm{nM} C X-5461\left(\mathrm{Gl} 25-\mathrm{Gl}_{50}\right.$ dose for proliferation at 2 days (Table S7)). At $48 \mathrm{~h}$ post transfection, cells were fixed, DAPI stained and imaged by high content microscopy to determine cell number. $\mathbf{b}$ Gene ontology analysis of hits identified in the primary screen. Significant enriched functional processes $(P$ value $<0.01$ ) associated with the primary screen hits. c Heatmap of the frequency of primary hits involved in DNA repair pathways. $\mathbf{d}$ Table of confident hits identified in the secondary deconvolution screen. e STRING network of the 20 validated screen hits. $\mathbf{f}$ Plot of Coefficient of drug interaction for individual siRNA duplexes against RAD54L, BRCA2, RAD51AP1 and TOP1 genes, modified from ref. ${ }^{15}$ (CC BY-NC 4.0).

the G2/M cell cycle checkpoint response to CX-5461 plus topotecan. To test this hypothesis, we first assessed DDR activation in OVCAR4, OVCAR3 and CAOV3 cells upon vehicle, CX-5461, topotecan or combination treatment for 3 or $24 \mathrm{~h}$ using Western blotting of phosphorylated checkpoint kinase 1 (CHK1) and CHK2 (downstream effectors of ATR and ATM, respectively), phospho-replication protein A (RPA) (Ser4/Ser8) and phospho-RPA (Ser33), which protect single-stranded DNA (ssDNA) (markers of replication stress), and $\mathrm{yH} 2 \mathrm{AX}$ (Ser139) (a marker of DNA damage) (Fig. 2c). Given recent reports identifying CX-5461 as a potential TOP2 poison, ${ }^{16,31,32}$ we also compared the response to the treatments with that of the TOP2 inhibitor doxorubicin. We observed increased phospho-CHK1 and phospho-CHK2 within $3 \mathrm{~h}$ upon treatment with CX-5461, topotecan or the combination compared to vehicle. At $24 \mathrm{~h}$ post treatment, in addition to increased activation of phospho-CHK1 and phospho-CHK2, among all three cell lines we consistently found increased phospho-RPA (Ser4/Ser8) and phospho-RPA (Ser33) with the combination treatment to the same level as that of doxorubicin compared to single-agent treatment, indicating significant induction of replication stress; however, we did not observe dramatic changes in global $\mathrm{\gamma H} 2 \mathrm{AX}$ (Ser139) by comparison to doxorubicin treatment (Fig. 3c).

As we have demonstrated, CX-5461 alone induces the formation of ssDNA and recruitment of phosphorylated RPA to the nucleolar periphery (the site of rDNA transcription), ${ }^{15}$ we first examined nucleolar localisation of phosphorylated RPA (Ser33) (Fig. 3a), a marker of stalled replication forks. ${ }^{33}$ We found a robust increase in phospho-RPA (Ser33) recruitment to the nucleoli marked by upstream binding transcription factor staining with the combination compared to single-agent treatment at $3 \mathrm{~h}$, particularly in replicating cells marked by EdU incorporation (Fig. 3b). The increased phospho-RPA (Ser33) recruitment indicative of replication fork stalling was specific to nucleoli as we did not observe any changes in overall nuclear phospho-RPA (Ser33) intensity (Fig. 3c). In contrast, doxorubicin treatment resulted in a significant increase in phospho-RPA (Ser33) in nucleoli and across the genome. We also examined the localisation of phosphorylated ATR, which is recruited to RPA-coated ssDNA (Fig. 3d). Treatment with CX-5461 in combination with topotecan enhanced nucleolar 
a
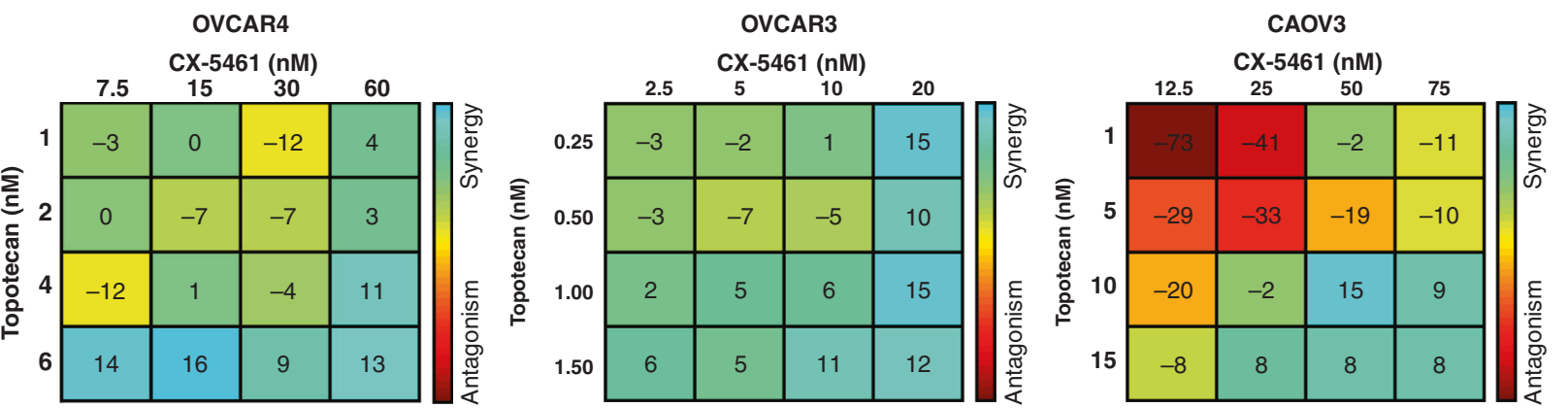

b

OVCAR4
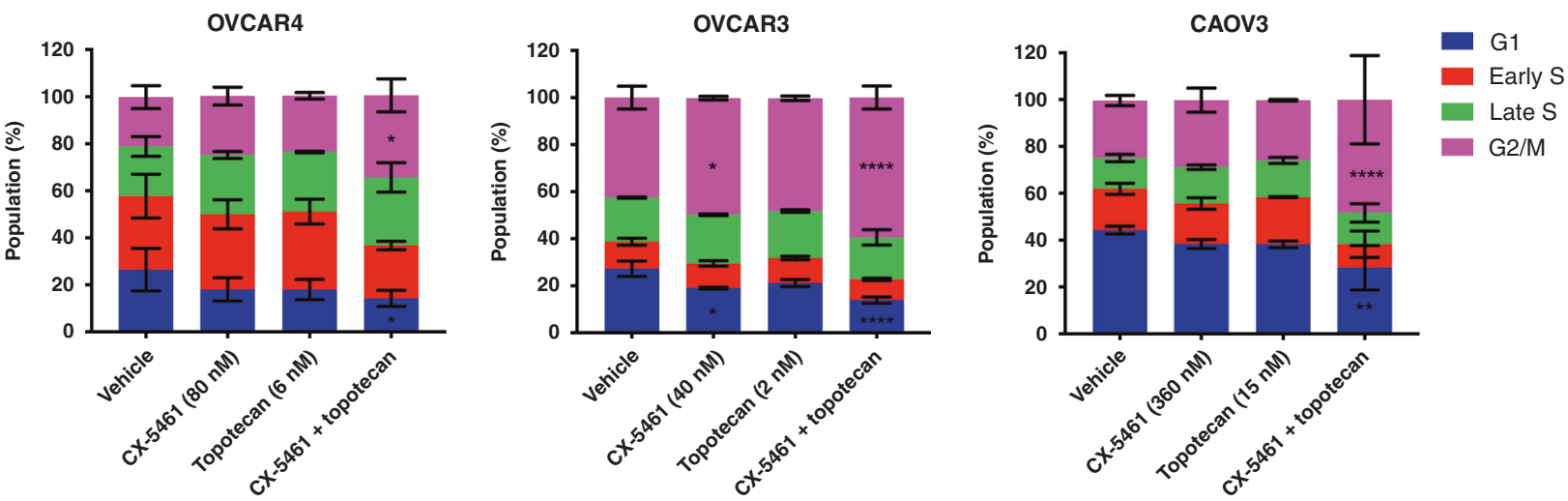

C
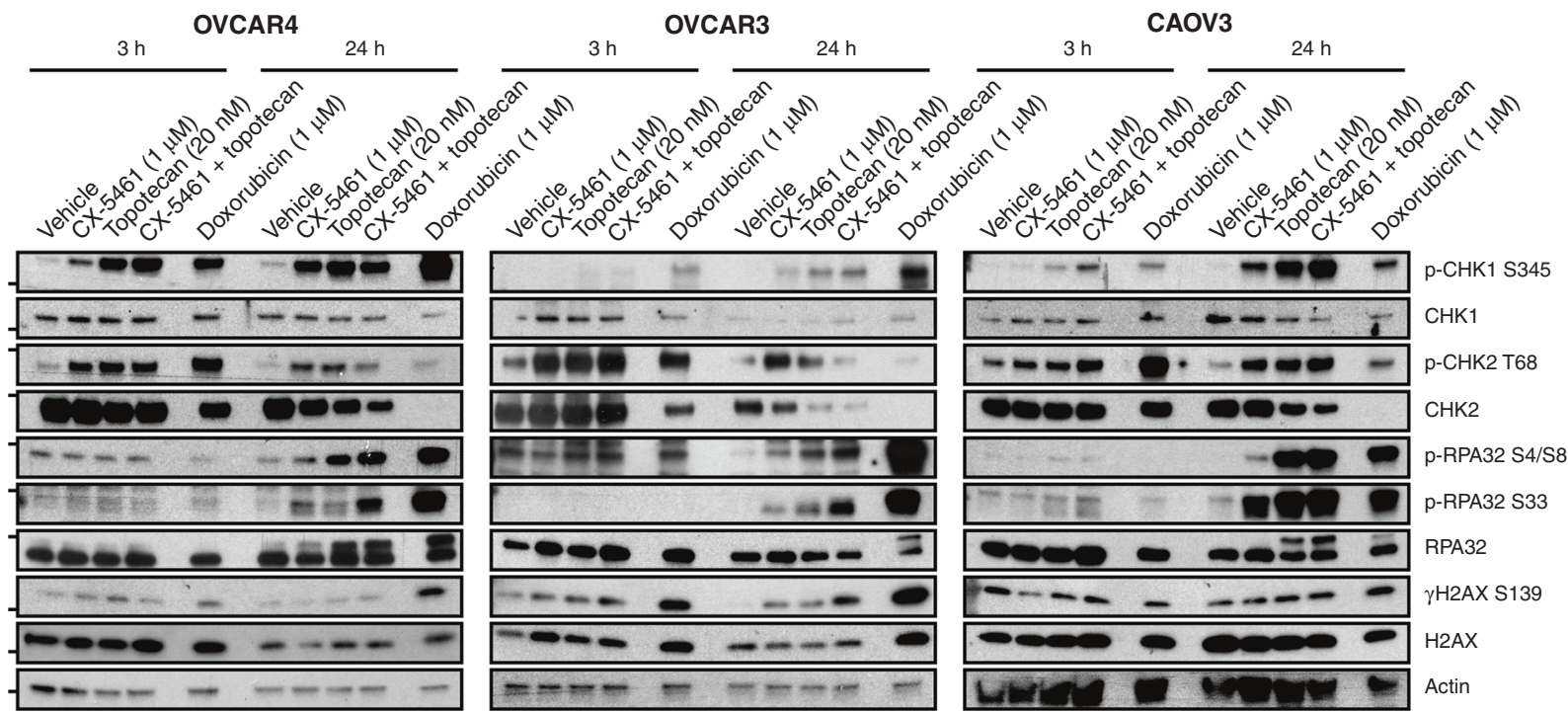

Fig. 2 The TOP1 inhibitor topotecan synergises with CX-5461 in multiple HR-proficient HGSC cell lines. a 2D mapped surface Combenefit BLISS plots show synergy scores for OVCAR4, OVCAR3 and CAOV3 cells treated in drug checkerboard assays with the indicated concentrations of CX-5461 and topotecan for 9 days. The range of concentrations for each drug was determined based on the $\mathrm{Gl}_{25}-\mathrm{Gl}_{50}$ dose at 5 days (Table S7). b Cell cycle analysis of OVCAR4, OVCAR3 and CAOV3 cells treated with vehicle, CX-5461 (80 nM, OVCAR4; 40 nM, OVCAR3; 360 nM, CAOV3; $\mathrm{Gl}_{50}$ for proliferation at $48 \mathrm{~h}$ ), topotecan $\left(6 \mathrm{nM}\right.$, OVCAR4; $2 \mathrm{nM}$, OVCAR3; $15 \mathrm{nM}, \mathrm{CAOV}_{3} ; \mathrm{Gl}_{50}$ for proliferation at $48 \mathrm{~h}$ ) or CX-5461 and topotecan. Quantification of the percentage of G1, early S, late $S$ and G2/M phase cells is presented as mean \pm SEM and statistical significance was determined by Kruskal-Wallis one-way ANOVA $\left({ }^{*} P<0.05 ;{ }^{* *} P<0.01 ;{ }^{* * *} P<0.001 ;{ }^{* * *} P<0.0001\right)$. c Representative Western blot analysis of DDR signalling in OVCAR4, OVCAR3 and CAOV3 cells treated with vehicle, $1 \mu \mathrm{M} \mathrm{CX}-5461,20 \mathrm{nM}$ topotecan, CX-5461 and topotecan or $1 \mu \mathrm{M}$ doxorubicin for 3 or $24 \mathrm{~h}$ ( $n=3$ experiments). The concentration for each drug was determined based on the TGI dose at 2 days. Actin was probed as a loading control.

(Fig. 3e) and global (Fig. 3f) phospho-ATR staining as compared to vehicle, although this was independent of DNA replication. Importantly, while nucleolar and global phospho-RPA (Ser33) was robustly induced upon doxorubicin treatment, phospho-ATR was not induced at this time point, indicating that the combination of CX-5461 and topotecan induces the DDR differently to doxorubicin. Together, these data demonstrate that CX-5461 plus topotecan induces the RPA/ATR/DDR axis and a robust $\mathrm{G} 2 / \mathrm{M}$ cell cycle arrest and cell death via a mechanism distinct from the TOP2 poison doxorubicin. 

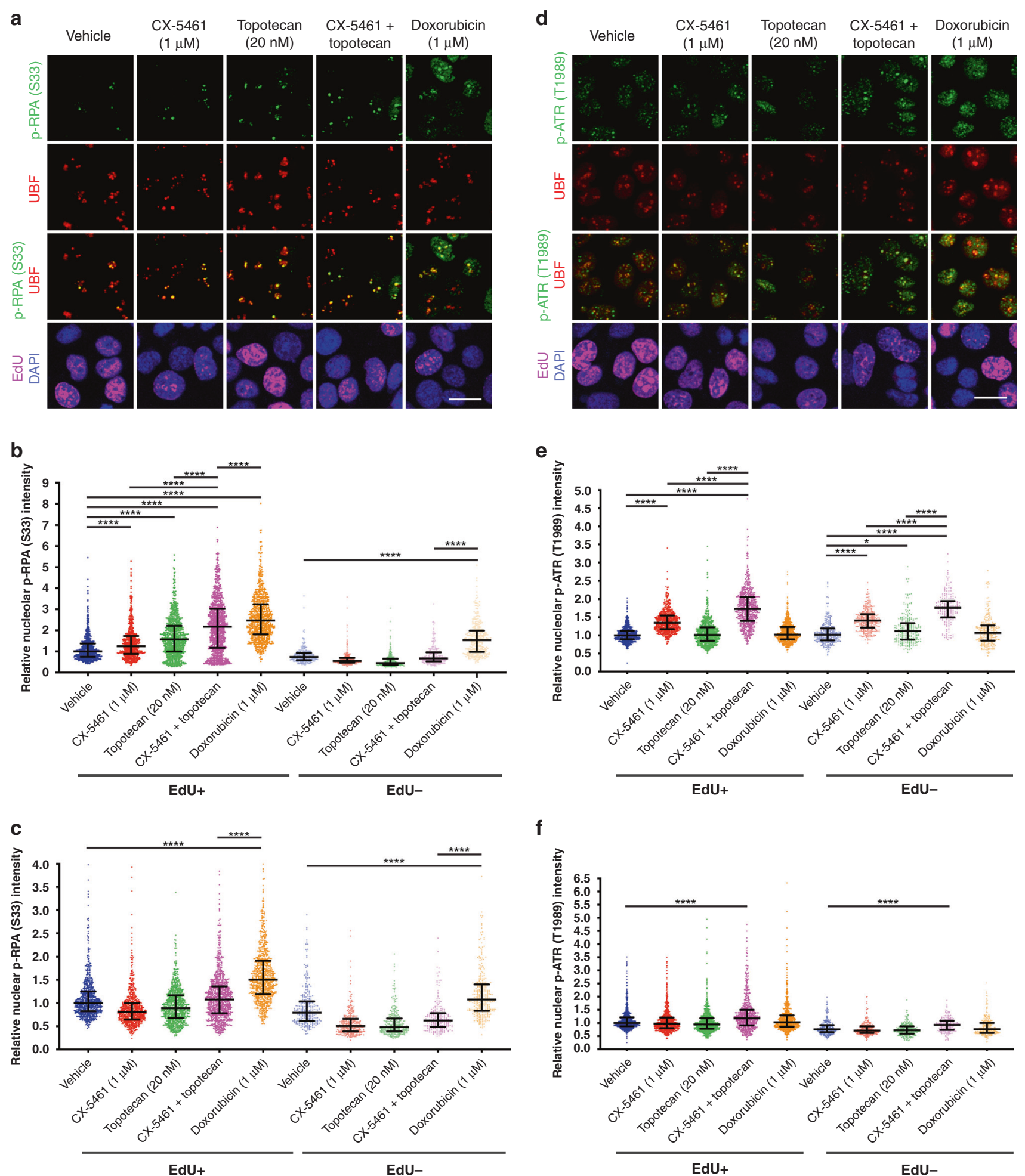

Fig. 3 CX-5461 plus topotecan potentiates the recruitment of phosphorylated RPA and ATR to nucleoli. a-f OVCAR4 cells were treated with vehicle, $1 \mu \mathrm{M}$ CX-5461, $20 \mathrm{nM}$ topotecan, CX-5461 and topotecan or $1 \mu \mathrm{M}$ doxorubicin for $3 \mathrm{~h}$. a, d Representative images and quantification of relative (b and $\mathbf{e}$ ) nucleolar or (c and $\mathbf{f}$ ) nuclear fluorescence intensity normalised to the Vehicle EdU $\mathbf{~}^{+}$median for $(\mathbf{b}$, $\mathbf{c}$ ) phospho-RPA (S33) or (e, f) phospho-ATR (T1989), respectively. Cells were stained for UBF, DAPI or EdU to label nucleoli, nuclei or replicating cells, respectively. Scale bar is $20 \mu \mathrm{m}$. Data are presented as median with interquartile range and statistical significance for increased p-RPA or p-ATR was determined by Kruskal-Wallis one-way ANOVA $\left({ }^{*} P<0.05 ;{ }^{* * * *} P<0.0001\right)$. 
a

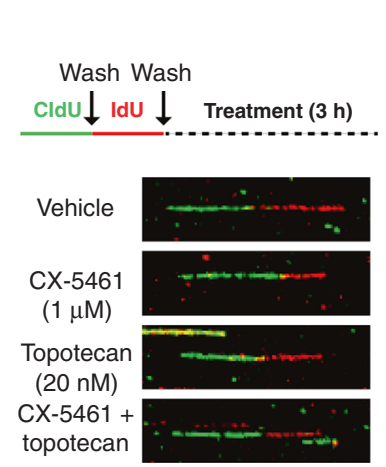

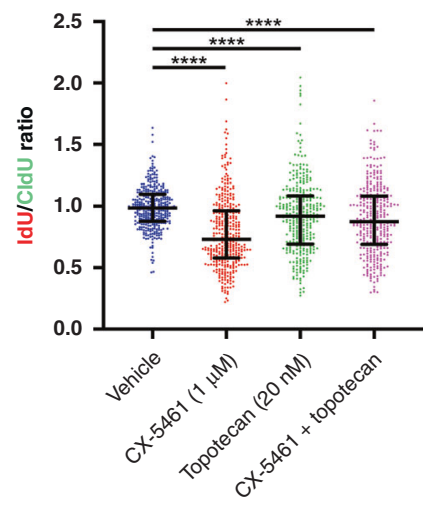

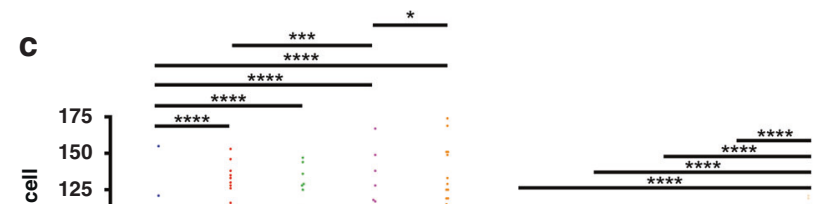

C

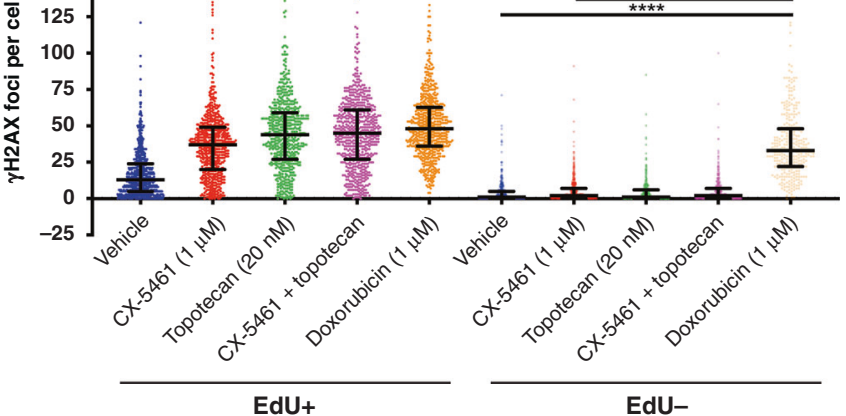

b

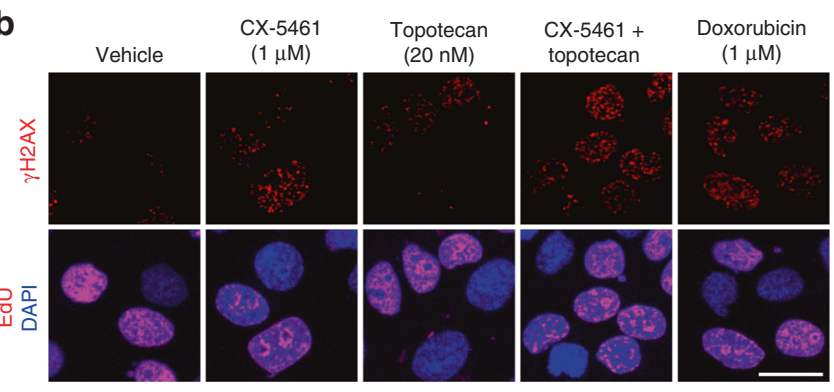

d

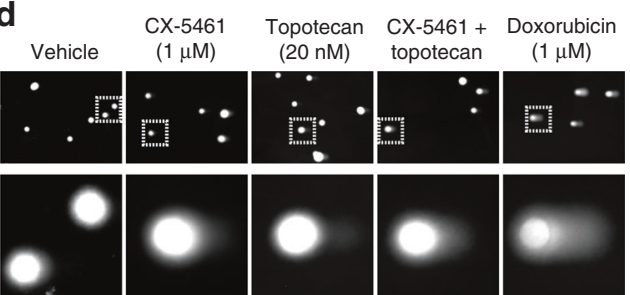

e

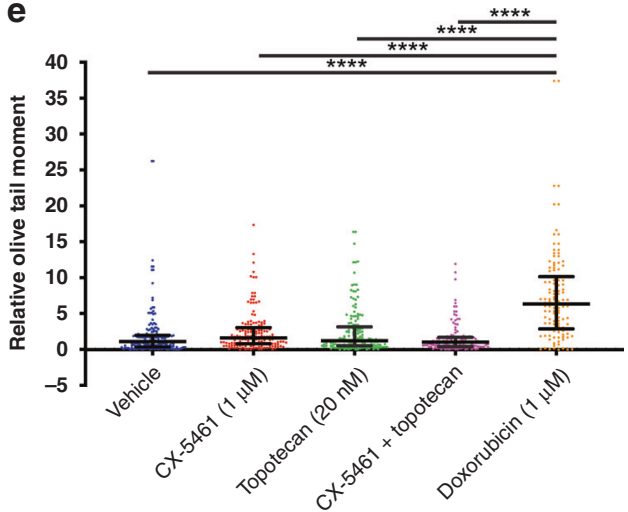

Fig. 4 The combination of CX-5461 and topotecan does not result in enhanced replication fork degradation or DNA strand break generation. a-e OVCAR4 cells were treated with $1 \mu \mathrm{M} \mathrm{CX}-5461,20 \mathrm{nM}$ topotecan, CX-5461 and topotecan or $1 \mu \mathrm{M}$ doxorubicin (as indicated) for $3 \mathrm{~h}$. a Representative images and quantification of decreased IdU to CldU ratio is represented as median with interquartile range and statistical significance was determined by Kruskal-Wallis one-way ANOVA (**** $P<0.0001$ ). b Representative images and $\mathbf{c}$ quantification of foci number for $\gamma \mathrm{H} 2 \mathrm{AX}$. Cells were stained for EdU and DAPI to label replicating cells and nuclei, respectively. Scale bar is $20 \mu \mathrm{m}$. Data are presented as median with interquartile range and statistical significance for increased foci per cell was determined by Kruskal-Wallis one-way ANOVA $\left({ }^{*} P<0.05 ;{ }^{* * *} P<0.001\right.$, $\left.{ }^{* * *} P<0.0001\right)$. d Representative images and e quantification of alkaline comet assay is presented as median with interquartile range and statistical significance was determined by Kruskal-Wallis one-way ANOVA ${ }^{* *} P<0.01 ;{ }^{* * * *} P<0.0001$ from $n=3$ experiments).

The combination of CX-5461 and topotecan enhances replication stress without inducing DNA strand breaks

We have shown that the CX-5461-mediated DDR is associated with degradation of stalled replication forks and replicationdependent $\gamma \mathrm{H} 2 \mathrm{AX}$ foci formation indicative of DNA damage in HGSC cells. ${ }^{15}$ To examine the impact of the combination of CX5461 and topotecan on replication fork stability, we performed DNA fibre analysis in OVCAR4 cells treated with vehicle, CX-5461, topotecan or the combination (Fig. 4a). In agreement with our previous observation in HR-proficient OVCAR8 cells, ${ }^{15}$ CX-5461 treatment-induced replication fork degradation in OVCAR4 cells, as indicated by a reduced IdU to CldU ratio. Topotecan also increased replication fork destabilisation compared to control. However, the combination of CX-5461 and topotecan did not result in enhanced fork degradation compared with either drug alone. To examine the persistence of stalled replication forks, we assessed the levels of phosphorylated RPA (Ser4/Ser8) upon treatment with vehicle, CX-5461, topotecan or the combination at $24 \mathrm{~h}$ (Fig. S4A). While we did not observe marked changes in nucleolar phospho-RPA (Ser4/Ser8) (Fig. S4B), we observed a significantly greater increase in nuclear Ser4/Ser8 phosphorylation of RPA in cells treated with the CX-5461 plus topotecan combination compared to single-agent therapy (Fig. S4C), indicating that the combination treatment leads to enhanced persistent replication stress. The data, therefore, suggest that the CX-5461 and topotecan combination enhances replication stress via different effects of each drug on fork progression and stability, possibly through fork degradation by CX-5461 and fork stalling by TOP1 trapping.

To examine the effect of combining CX-5461 and topotecan on DNA damage, we examined $\mathrm{YH} 2 \mathrm{AX}$ foci formation in OVCAR4 cells (Fig. 4b). Treatment with CX-5461, topotecan or the combination led to increased $\mathrm{\gamma H} 2 \mathrm{AX}$ foci formation compared to vehicle exclusively in S-phase EdU-positive cells (Fig. 4c). Interestingly, in EdU-positive cells treatment with the combination of $\mathrm{CX}-5461$ and topotecan led to $\mathrm{yH} 2 \mathrm{AX}$ foci formation almost to the same level as that observed upon doxorubicin treatment. However, doxorubicin treatment also induced $\mathrm{\gamma H} 2 \mathrm{AX}$ 
a

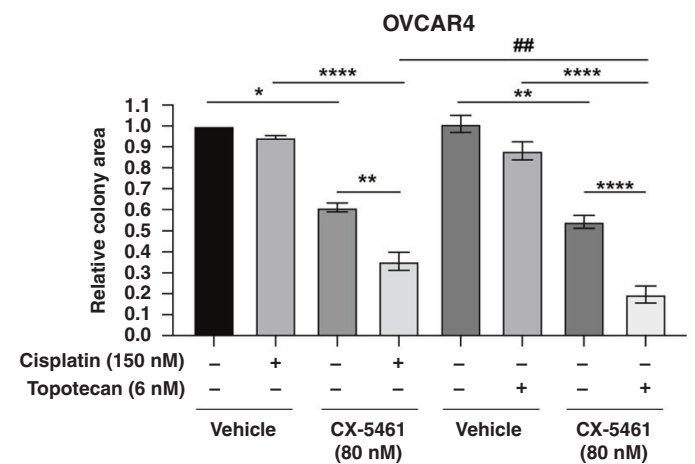

b

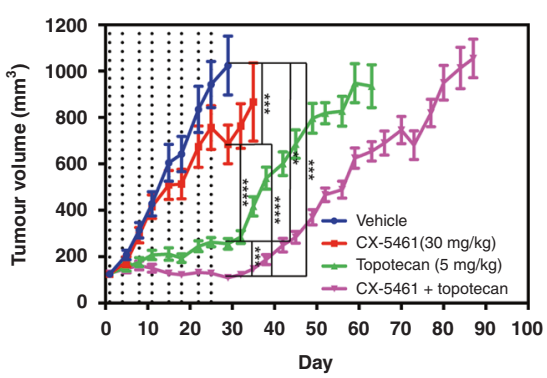

c

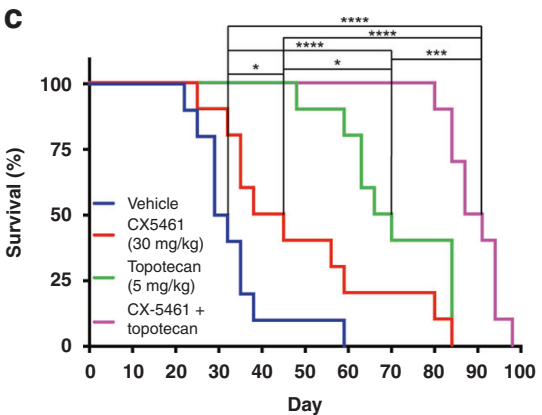

d

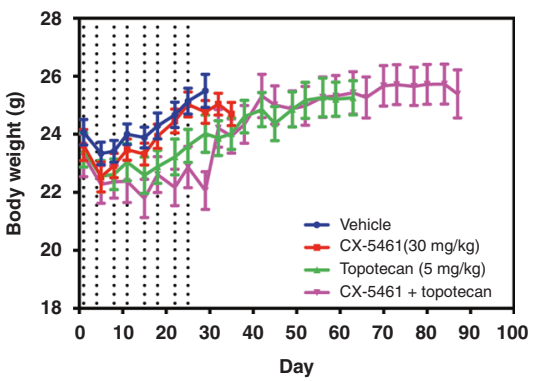

Fig. 5 CX-5461 plus topotecan inhibits HR-proficient HGSC clonogenic survival and tumour growth. a Clonogenic survival assays for OVCAR4 cells treated with vehicle or approximate $\mathrm{Gl}_{50}$ doses of CX-5461 in the absence or presence of the indicated concentrations of cisplatin or topotecan for $48 \mathrm{~h}$. Drugs were washed out and cells were cultured for an additional 5 days, fixed and stained with crystal violet. Relative colony area normalised to vehicle without cisplatin is presented as mean \pm SEM and statistical significance for the decrease in relative colony area was determined by one-way ANOVA $\left({ }^{*} P<0.05 ;{ }^{* *} P<0.01 ; * * * * P<0.0001 ; C X-5461\right.$ with cisplatin vs. CX-5461 with topotecan, ${ }^{\# \#} P<$ 0.01). b Mean tumour volume of OVCAR3 flank tumours treated with vehicle, CX-5461 (30 mg/kg), topotecan (5 mg/kg) or CX-5461 (30 mg/kg) and topotecan $(5 \mathrm{mg} / \mathrm{kg})(n=10$ mice/group). Data are presented as mean \pm SEM and statistical significance was determined on day 22 by one-way ANOVA (vehicle vs. CX-5461, topotecan or combination, CX-5461 vs. topotecan or combination, ${ }^{* * * * P<0.0001 ;}$ topotecan vs. combination, $\left.{ }^{* * *} P<0.001\right)$. c Kaplan-Meier survival curves of tumour-bearing mice. Statistical significance was determined by log-rank Mantel-Cox tests (vehicle vs. CX-5461, CX-5461 vs. topotecan, ${ }^{*} P<0.05$; vehicle vs. topotecan or combination, CX-5461 vs. combination, ${ }^{* * * *} P$ $<0.0001$; topotecan vs. combination, $\left.{ }^{* *} P<0.001\right)$. d Mouse body weight. Dashed line indicates day of drug dosing.

foci formation in EdU-negative cells, highlighting that activation of the DDR to the combination of CX-5461 and topotecan requires active DNA replication.

Replication stress due to stalled replication forks can induce ATR/ATM activation and $\mathrm{YH} 2 \mathrm{AX}$ prior to the formation of DNA strand breaks. ${ }^{34}$ Furthermore, although phosphorylated H2AX is considered to be a marker of DNA damage, it does not always equate to DNA strand breakage. ${ }^{35}$ We performed comet assays of OVCAR4 cells after $3 \mathrm{~h}$ of treatment with vehicle, CX-5461, topotecan or the combination to test if the treatments that induced $\mathrm{\gamma H} 2 \mathrm{AX}$ foci levels were associated with DNA strand breaks (Fig. 4d). Alkaline comet assays, which can detect singlestrand breaks, DSBs, DNA cross-linking and alkali labile sites, ${ }^{36}$ revealed that CX-5461 and topotecan alone or in combination did not induce comet tails. This was in striking contrast to doxorubicin, which induced prominent comet tails indicating extensive DNA breakage (Fig. 4e). Thus, the enhanced replication stress by the CX-5461 plus topotecan combination does not generate DNA strand breaks, which may be beneficial in reducing any potential genotoxicity of this therapeutic approach.

CX-5461 plus topotecan inhibits clonogenic survival and blocks tumour growth

We have demonstrated recently that CX-5461 acts to overcome fork protection in olaparib-resistant HGSC cells. ${ }^{15}$ Cells resistant to PARP inhibitors are also tolerant to topotecan and cisplatin, indicating that stalled fork protection and stabilisation confer a general resistance to replication stress-inducing chemotherapeutics. 3,4 Nevertheless, to compare the cellular response of the CX-5461 plus topotecan combination therapy with standard HGSC platinum-based therapy, we assessed colony formation of OVCAR4 cisplatin-sensitive, olaparibresistant cells (Fig. S2C, D) treated with vehicle, cisplatin, CX5461, topotecan, CX-5461 plus cisplatin or CX-5461 plus topotecan, followed by drug washout (Fig. 5a). While we found a decrease in colony formation due to each drug treatment alone compared to control (Fig. 5a), the decrease in colony formation was significantly more robust with CX-5461 plus cisplatin or CX-5461 plus topotecan compared to single-agent treatment. Moreover, the combination of CX-5461 and topotecan led to a significantly greater decrease in colony formation when compared with CX-5461 plus cisplatin, highlighting CX5461 plus topotecan as a promising alternative to standard chemotherapy.

To determine the impact on tumour growth in vivo, we established tumours from HR-proficient OVCAR3 cells harbouring amplified CCNE1, which confers primary treatment resistance and poor outcome of HGSC. ${ }^{8}$ We assessed tumour growth (Fig. 5b) and survival (Fig. $5 \mathrm{C}$ ) in response to treatment with CX-5461 (30 mg/ $\mathrm{kg}$ ), topotecan (5 mg/kg) or CX-5461 plus topotecan. We chose a standard topotecan dose of $5 \mathrm{mg} / \mathrm{kg}$, which is well below the dose reported to potentially result in haematologic toxicity $(12.5 \mathrm{mg} /$ $\mathrm{kg}){ }^{37}$ Mice were dosed twice weekly for 4 weeks and then monitored until the animals reached an ethical endpoint. CX-5461 or topotecan treatment alone delayed tumour growth and 
prolonged survival as compared to vehicle treatment. Strikingly, the combined treatment of CX-5461 and topotecan blocked tumour progression during the entirety of drug treatment, significantly extending survival. The combination was well tolerated with a mean weight loss $<10 \%$ (Fig. $5 \mathrm{~d}$ ). Taken together, these data demonstrate that the combination of CX-5461 and topotecan robustly inhibits the growth of HR-proficient HGSC cells and tumours.

\section{DISCUSSION}

We and others have identified a new paradigm for treating oncogene-driven cancers by targeting ribosome biogenesis. ${ }^{10,11,13-15,26,29,38-40}$ We have demonstrated that CX5461 can inhibit Pol I recruitment to $\mathrm{rDNA}^{10}{ }^{10}$ induce a ribosome biogenesis checkpoint in cells with intact $\mathrm{p} 53^{13}$ and activate a p53independent DDR. ${ }^{29}$ We have also exploited CX-5461's ability to activate the p53-independent DDR and its different sensitivity profile to standard therapies to target HGSC. ${ }^{15}$ CX-5461 is synthetic lethal with HR deficiency in vitro and in vivo in HGSC via a mechanism that is distinct from PARP inhibitors. ${ }^{15}$ Here, we found TOP1 inhibition, which has been shown to be effective in HRdeficient disease, ${ }^{41}$ can cooperate with CX-5461 in HR-proficient HGSC. Thus, our findings can potentially expand the application of both CX-5461 and TOP1 inhibitors such as topotecan in clinical trials to the $50 \%$ of HGSC patients who have HR-proficient tumours and to patients with HR-deficient tumours that have acquired chemotherapeutic resistance due to restored HR.

Recently, several studies have identified that CX-5461 has a similar sensitivity profile to DNA TOP2 poisons ${ }^{16,31,32}$ and requires TOP2 for cytotoxicity. ${ }^{31,32}$ TOP $2 a$ is a component of the RNA polymerase I pre-initiation complex ${ }^{42}$ and it is possible that CX-5461's ability to trap TOP2 can contribute to its 200-fold selectivity for Pol I transcription inhibition over the other RNA polymerases $^{10,43}$ as well as its therapeutic efficacy across the genome. In contrast, through an independent approach utilising a genome-wide RNAi screen, we show CX-5461 has a different sensitivity profile to those recently reported for TOP2 poisons (Fig. 1c). ${ }^{16,31,32}$ We have identified that TOP1 inhibition synergises with CX-5461 to inhibit HR-proficient HGSC cell proliferation, a critical finding supported by other studies demonstrating decreased survival upon treatment with pyridostatin in HeLa cells ${ }^{32}$ or with CX-5461 in E $\mu-M y c$ lymphoma $^{31}$ depleted of TOP1. Both TOP1 and TOP2 can localise to rDNA and are involved in regulating torsional stress during transcription and DNA replication. ${ }^{18,42}$ However, the differences in how they do so could explain why deficiencies in TOP1 and TOP2 confer sensitivity and resistance, respectively, to CX-5461. Indeed, a report in yeast has shown that TOP1 plays a critical role behind elongating RNA Pol I, while TOP2 plays a more critical role in front of elongating Pol I. ${ }^{44}$ In addition, G-quadruplex stabilisers such as pyridostatin can induce TOP2-dependent DNA DSBs that are countered by TOP1, likely through TOP1's ability to regulate negative supercoiling behind RNA Pol I. ${ }^{32}$ With the combination of CX-5461 and the TOP1 inhibitor topotecan in HR-proficient HGSC cells, we found robust nucleolar recruitment of phosphorylated RPA (Ser33) and ATR. We previously found that CX5461 enhances rDNA chromatin accessibility to MNase. ${ }^{29}$ Thus, we hypothesise that topotecan's ability to enhance negative torsional stress combined with increased chromatin accessibility upon treatment with CX-5461 contributes to enhanced nucleolar recruitment of activated RPA and ATR, leading to a robust nucleolar and global DDR in a manner distinct to the TOP2 poison doxorubicin. Future studies utilising chromatin immunoprecipitation and electron microscopy of TOP1 and TOP2 at rDNA will be important to further elucidate the spatial mechanisms by which TOP1 and TOP 2 confer sensitivity and resistance to CX-5461.
Intriguingly, we found either depletion of TOP1 or treatment with the TOP1 inhibitor topotecan could enhance the antiproliferative response to CX-5461 in HR-proficient HGSC. Previous studies have shown that TOP1 deficiency can result in the accumulation of stalled replication forks ${ }^{45,46}$ and sublethal doses of TOP1 inhibitors can slow replication fork progression and induce reversed forks. ${ }^{47,48}$ We have also shown that CX-5461 induces MRE11-dependent degradation of replication forks. ${ }^{15}$ Therefore, we propose that fork degradation by CX-5461 and fork stalling by TOP1 depletion/inhibition together contribute to enhanced persistent replication stress. Overall, the combination of CX-5461 and topotecan leads to enhanced replication stress and DDR without eliciting DNA breakage, highlighting a lower potential for genotoxicity that normally leads to adverse side effects from damaging normal cells. Nonetheless, even in the absence of DNA breakage, the combination promotes a robust G2/M arrest that impairs clonogenic survival and maintains potent anti-tumour activity.

As a complement to our previous findings that CX-5461 has efficacy in HGSC harbouring HR deficiency and high MYC activity driving Pol I transcription and/or MYC-driven global transcription and replication stress, here we identify a potent anti-proliferative combination between CX-5461 and TOP1 inhibition in HRproficient HGSC. We show that CX-5461 and the TOP1 inhibitor topotecan cooperate by enhancing DDR and replication stress without generating DNA strand breaks, leading to a robust G2/M cell cycle arrest, inhibition of clonogenic survival and tumour growth in vivo. Topotecan as a salvage therapy is used at maximum-tolerated doses, which can cause myelosuppression that limits its use clinically. However, we found that using lowdose topotecan cooperates with CX-5461. We suggest that further investigation into modifying dosing strategies with TOP1 inhibitors such as topotecan will facilitate using this class of drugs in combination with CX-5461 as a promising therapeutic option for HGSC patients.

\section{ACKNOWLEDGEMENTS}

We thank Daniel Thomas, Jennii Luu, Jessica Ahern, Kerry Ardley and Rachael Walker for their technical support. The original submitted version of this manuscript was deposited on the preprint server bioRxiv. ${ }^{49}$

\section{AUTHOR CONTRIBUTIONS}

K.T.C., R.B.P. and E.S. conceived the project. S.Y. drafted the manuscript and K.T.C., R.B. P. and E.S. wrote the paper. S.E., K.J.S., J.K., C.C., K.E.S., K.M.H. and R.D.H. provided intellectual input and revised the manuscript. S.Y., J.X., N.B., M.R.C.T and K.T.C. performed the experiments. P.B.M. analysed the genome-wide RNAi screen data. Animal studies were performed in collaboration with C.C. The screen was a collaboration with K.J.S.

\section{ADDITIONAL INFORMATION}

Ethics approval and consent to participate All animal experiments were performed in accordance with the Animal Experimentation Ethics Committee guidelines (Protocol \#E557) at the Peter MacCallum Cancer Centre, Australia.

Data availability The datasets are presented in the additional supporting files.

Competing interests There are no competing financial interests in relation to the work described.

Funding information The China Scholarship Council University of Melbourne Ph.D. Scholarship supported S.Y. A National Health and Medical Research Council (NHMRC) Grant and NHMRC Senior Research Fellowship to R.B.P. supported this work. The Victorian Centre for Functional Genomics (K.J.S.) is funded by the Australian Cancer Research Foundation (ACRF), the Australian Phenomics Network (APN) through funding from the Australian Government's National Collaborative Research Infrastructure Strategy (NCRIS) programme, the Peter MacCallum Cancer Centre 
Foundation and the University of Melbourne Research Collaborative Infrastructure Programme.

Supplementary information is available for this paper at https://doi.org/10.1038/ s41416-020-01158-z.

Publisher's note Springer Nature remains neutral with regard to jurisdictional claims in published maps and institutional affiliations.

\section{REFERENCES}

1. Konstantinopoulos, P. A., Ceccaldi, R., Shapiro, G. I. \& D'Andrea, A. D. Homologous recombination deficiency: exploiting the fundamental vulnerability of ovarian cancer. Cancer Discov. 5, 1137-1154 (2015).

2. Ashworth, A. \& Lord, C. J. Synthetic lethal therapies for cancer: what's next after PARP inhibitors? Nat. Rev. Clin. Oncol. 15, 564-576 (2018)

3. Chaudhuri, A. R., Callen, E., Ding, X., Gogola, E., Duarte, A. A., Lee, J. E. et al. Replication fork stability confers chemoresistance in BRCA-deficient cells. Nature 535, 382-387 (2016)

4. Chaudhuri, A. R., Callen, E., Ding, X., Gogola, E., Duarte, A. A., Lee, J.-E. et al. Erratum: replication fork stability confers chemoresistance in BRCA-deficient cells. Nature 539, 456 (2016).

5. Christie, E. L., Fereday, S., Doig, K., Pattnaik, S., Dawson, S. J. \& Bowtell, D. D. L. Reversion of BRCA1/2 germline mutations detected in circulating tumor DNA from patients with high-grade serous ovarian cancer. J. Clin. Oncol. 35, 1274-1280 (2017)

6. Kondrashova, O., Nguyen, M., Shield-Artin, K., Tinker, A. V., Teng, N. N. H., Harrell, M. I. et al. Secondary somatic mutations restoring RAD51C and RAD51D associated with acquired resistance to the PARP inhibitor rucaparib in high-grade ovarian carcinoma. Cancer Discov. 7, 984-998 (2017).

7. Wakefield, M. J., Nesic, K., Kondrashova, O. \& Scott, C. L. Diverse mechanisms of PARP inhibitor resistance in ovarian cancer. Biochim. Biophys. Acta Rev. Cancer 1872, 188307 (2019)

8. Au-Yeung, G., Lang, F., Azar, W. J., Mitchell, C., Jarman, K. E., Lackovic, K. et al. Selective targeting of cyclin E1-amplified high-grade serous ovarian cancer by cyclin-dependent kinase 2 and AKT inhibition. Clin. Cancer Res. 23, 1862-1874 (2017).

9. Yan, S., Frank, D., Son, J., Hannan, K. M., Hannan, R. D., Chan, K. T. et al. The potential of targeting ribosome biogenesis in high-grade serous ovarian cancer. Int. J. Mol. Sci. 18, 210 (2017).

10. Drygin, D., Lin, A., Bliesath, J., Ho, C. B., O'Brien, S. E., Proffitt, C. et al. Targeting RNA polymerase I with an oral small molecule CX-5461 inhibits ribosomal RNA synthesis and solid tumor growth. Cancer Res. 71, 1418-1430 (2011).

11. Khot, A., Brajanovski, N., Cameron, D. P., Hein, N., Maclachlan, K. H., Sanij, E. et al. First-in-human RNA polymerase I transcription inhibitor CX-5461 in patients with advanced hematologic cancers: results of a phase I dose-escalation study. Cancer Discov. 9, 1036-1049 (2019).

12. Hilton, J., Cescon, D., Bedard, P., Ritter, H., Tu, D., Soong, J. et al. 440 CCTG IND. 231: a phase 1 trial evaluating CX-5461 in patients with advanced solid tumors. Ann. Oncol. 29(Suppl. 3), iii8 (2018).

13. Bywater, M. J., Poortinga, G., Sanij, E., Hein, N., Peck, A., Cullinane, C. et al. Inhibition of RNA polymerase I as a therapeutic strategy to promote cancer-specific activation of p53. Cancer Cell 22, 51-65 (2012).

14. Devlin, J. R., Hannan, K. M., Hein, N., Cullinane, C., Kusnadi, E., Ng, P. Y et al. Combination therapy targeting ribosome biogenesis and mRNA translation synergistically extends survival in MYC-driven lymphoma. Cancer Discov. 6, 59-70 (2016).

15. Sanij, E., Hannan, K. M., Xuan, J., Yan, S., Ahern, J. E., Trigos, A. S. et al. CX-5461 activates the DNA damage response and demonstrates therapeutic efficacy in high-grade serous ovarian cancer. Nat. Commun. 11, 2641 (2020).

16. Olivieri, M., Cho, T., Alvarez-Quilon, A., Li, K., Schellenberg, M. J., Zimmermann, M. et al. A genetic map of the response to DNA damage in human cells. Cell 182, 481-496 (2020).

17. Koster, D. A., Croquette, V., Dekker, C., Shuman, S. \& Dekker, N. H. Friction and torque govern the relaxation of DNA supercoils by eukaryotic topoisomerase IB. Nature 434, 671-674 (2005)

18. Muller, M. T., Pfund, W. P., Mehta, V. B. \& Trask, D. K. Eukaryotic type I topoisomerase is enriched in the nucleolus and catalytically active on ribosomal DNA. EMBO J. 4, 1237-1243 (1985).

19. Zimmer, J., Tacconi, E. M. C., Folio, C., Badie, S., Porru, M., Klare, K. et al. Targeting BRCA1 and BRCA2 Deficiencies with G-quadruplex-interacting compounds. Mol. Cell 61, 449-460 (2016).
20. Bliss, C. The toxicity of poisons applied jointly 1. Ann. Appl Biol. 26, 585-615 (1939).

21. Schindelin, J., Arganda-Carreras, I., Frise, E., Kaynig, V., Longair, M., Pietzsch, T. et al. Fiji: an open-source platform for biological-image analysis. Nat. Methods 9, 676-682 (2012)

22. Carpenter, A. E., Jones, T. R., Lamprecht, M. R., Clarke, C., Kang, I. H., Friman, O. et al. CellProfiler: image analysis software for identifying and quantifying cell phenotypes. Genome Biol. 7, R100 (2006).

23. Gyori, B. M., Venkatachalam, G., Thiagarajan, P. S., Hsu, D. \& Clement, M. V. OpenComet: an automated tool for comet assay image analysis. Redox Biol. 2, 457-465 (2014).

24. Di Veroli, G. Y., Fornari, C., Wang, D., Mollard, S., Bramhall, J. L., Richards, F. M. et al. Combenefit: an interactive platform for the analysis and visualization of drug combinations. Bioinformatics 32, 2866-2868 (2016).

25. Domcke, S., Sinha, R., Levine, D. A., Sander, C. \& Schultz, N. Evaluating cell lines as tumour models by comparison of genomic profiles. Nat. Commun. 4, 2126 (2013).

26. Xu, H., Di Antonio, M., McKinney, S., Mathew, V., Ho, B., O'Neil, N. J. et al. CX-5461 is a DNA G-quadruplex stabilizer with selective lethality in BRCA1/2 deficient tumours. Nat. Commun. 8, 14432 (2017).

27. McGuire, W. P., Blessing, J. A., Bookman, M. A., Lentz, S. S. \& Dunton, C. J. Topotecan has substantial antitumor activity as first-line salvage therapy in platinum-sensitive epithelial ovarian carcinoma: a Gynecologic Oncology Group study. J. Clin. Oncol. 18, 1062-1062 (2000).

28. Gordon, A. N., Fleagle, J. T., Guthrie, D., Parkin, D. E., Gore, M. E. \& Lacave, A. J. Recurrent epithelial ovarian carcinoma: a randomized phase III study of pegylated liposomal doxorubicin versus topotecan. J. Clin. Oncol. 19, 3312-3322 (2001).

29. Quin, J., Chan, K. T., Devlin, J. R., Cameron, D. P., Diesch, J., Cullinane, C. et al. Inhibition of RNA polymerase I transcription initiation by CX-5461 activates noncanonical ATM/ATR signaling. Oncotarget 7, 49800-49818 (2016).

30. Marechal, A. \& Zou, L. DNA damage sensing by the ATM and ATR kinases. Cold Spring Harb. Perspect. Biol. 5, a012716 (2013).

31. Bruno, P. M., Lu, M., Dennis, K. A., Inam, H., Moore, C. J., Sheehe, J. et al. The primary mechanism of cytotoxicity of the chemotherapeutic agent CX-5461 is topoisomerase II poisoning. Proc. Natl Acad. Sci. USA 117, 4053-4060 (2020).

32. Pipier, A., Bossaert, M., Riou, J. F., Noirot, C., Nguyễn, L., Serre, R. F. et al. Transcription-associated topoisomerase activities control DNA-breaks production by G-quadruplex ligands. Preprint at https://www.biorxiv.org/content/10.1101/ 2020.02.18.953851v1 (2020).

33. Vassin, V. M., Anantha, R. W., Sokolova, E., Kanner, S. \& Borowiec, J. A. Human RPA phosphorylation by ATR stimulates DNA synthesis and prevents ssDNA accumulation during DNA-replication stress. J. Cell Sci. 122, 4070-4080 (2009).

34. Burgess, R. C. \& Misteli, T. Not all DDRs are created equal: non-canonical DNA damage responses. Cell 162, 944-947 (2015)

35. Tu, W.-Z., Li, B., Huang, B., Wang, Y., Liu, X.-D., Guan, H. et al. h2AX foci formation in the absence of DNA damage: mitotic $\mathrm{H} 2 \mathrm{AX}$ phosphorylation is mediated by the DNA-PKcs/CHK2 pathway. FEBS Lett. 587, 3437-3443 (2013).

36. Hartmann, A., Agurell, E., Beevers, C., Brendler-Schwaab, S., Burlinson, B., Clay, P. et al. Recommendations for conducting the in vivo alkaline Comet assay. 4th International Comet Assay Workshop. Mutagenesis 18, 45-51 (2003).

37. Guichard, S., Montazeri, A., Chatelut, E., Hennebelle, I., Bugat, R. \& Canal, P. Schedule-dependent activity of topotecan in OVCAR-3 ovarian carcinoma xenograft: pharmacokinetic and pharmacodynamic evaluation. Clin. Cancer Res 7, 3222-3228 (2001)

38. Hein, N., Cameron, D. P., Hannan, K. M., Nguyen, N. N., Fong, C. Y., Sornkom, J. et al. Inhibition of Pol I transcription treats murine and human AML by targeting the leukemia-initiating cell population. Blood 129, 2882-2895 (2017).

39. Ismael, M., Webb, R., Ajaz, M., Kirkby, K. J. \& Coley, H. M. The targeting of RNA polymerase I transcription using CX-5461 in combination with radiation enhances tumour cell killing effects in human solid cancers. Cancers 11, 1429 (2019).

40. Rebello, R. J., Kusnadi, E., Cameron, D. P., Pearson, H. B., Lesmana, A., Devlin, J. R. et al. The dual inhibition of RNA Pol I transcription and PIM kinase as a new therapeutic approach to treat advanced prostate cancer. Clin. Cancer Res 22, 5539-5552 (2016).

41. Maede, Y., Shimizu, H., Fukushima, T., Kogame, T., Nakamura, T., Miki, T. et al. Differential and common DNA repair pathways for topoisomerase I- and IItargeted drugs in a genetic DT40 repair cell screen panel. Mol. Cancer Ther. 13, 214-220 (2014).

42. Ray, S., Panova, T., Miller, G., Volkov, A., Porter, A. C., Russell, J. et al. Topoisomerase llalpha promotes activation of RNA polymerase I transcription by facilitating pre-initiation complex formation. Nat. Commun. 4, 1598 (2013).

43. Haddach, M., Schwaebe, M. K., Michaux, J., Nagasawa, J., O’Brien, S. E., Whitten, J. P. et al. Discovery of CX-5461, the First Direct and Selective Inhibitor of RNA Polymerase I, for Cancer Therapeutics. ACS Med. Chem. Lett. 3, 602-606 (2012). 
The RNA polymerase I transcription inhibitor CX-5461 cooperates with... $S$ Yan et al.

44. French, S. L., Sikes, M. L., Hontz, R. D., Osheim, Y. N., Lambert, T. E., El Hage, A. et al. Distinguishing the roles of Topoisomerases I and II in relief of transcription-induced torsional stress in yeast rRNA genes. Mol. Cell Biol. 31, 482-494 (2011).

45. Miao, Z. H., Player, A., Shankavaram, U., Wang, Y. H., Zimonjic, D. B., Lorenzi, P. L. et al. Nonclassic functions of human topoisomerase I: genome-wide and pharmacologic analyses. Cancer Res. 67, 8752-8761 (2007).

46. Tuduri, S., Crabbé, L., Conti, C., Tourrière, H., Holtgreve-Grez, H., Jauch, A. et al. Topoisomerase I suppresses genomic instability by preventing interference between replication and transcription. Nat. Cell Biol. 11, 1315-1324 (2009).

47. Chaudhuri, A. R., Hashimoto, Y., Herrador, R., Neelsen, K. J., Fachinetti, D., Bermejo, R. et al. Topoisomerase I poisoning results in PARP-mediated replication fork reversal. Nat. Struct. Mol. Biol. 19, 417 (2012).

48. Zellweger, R., Dalcher, D., Mutreja, K., Berti, M., Schmid, J. A., Herrador, R. et al. Rad51-mediated replication fork reversal is a global response to genotoxic treatments in human cells. J. Cell Biol. 208, 563-579 (2015).

49. Yan, S., Madhamshettiwar, P. B., Simpson, K. J., Ellis, S., Kang, J., Cullinane, C. et al. Targeting RNA polymerase I transcription synergises with TOP1 inhibition in potentiating the DNA damage response in high-grade serous ovarian cancer. Preprint at https://www.biorxiv.org/content/10.1101/849307v1 (2019).

(i) Open Access This article is licensed under a Creative Common Attribution 4.0 International License, which permits use, sharing, adaptation, distribution and reproduction in any medium or format, as long as you give appropriate credit to the original author(s) and the source, provide a link to the Creative Commons license, and indicate if changes were made. The images or other third party material in this article are included in the article's Creative Commons license, unless indicated otherwise in a credit line to the material. If material is not included in the article's Creative Commons license and your intended use is not permitted by statutory regulation or exceeds the permitted use, you will need to obtain permission directly from the copyright holder. To view a copy of this license, visit http://creativecommons. org/licenses/by/4.0/.

(c) The Author(s) 2020 